\title{
Wear Analysis of Discs and Balls on a Micro-Scale
}

\author{
Pawel PAWLUS, Andrzej DZIERWA
}

\begin{abstract}
Surface topographies of discs and balls after wear process were analysed. Discs were made from $42 \mathrm{CrMo} 4$ steel with hardness of $42 \mathrm{HRC}$, but balls from $100 \mathrm{Cr} 6$ steel of hardness $64 \mathrm{HRC}$. They were measured using white light interferometer Talysurf $\mathrm{CCl}$ Lite. Procedures for minimizing errors of wear loss determination were discussed. They can be applied thanks to software TalyMap. It was found that application of wear analysis of discs and balls on a micro scale allowed us precise determination of their wear after tribological tests, however this analysis should be careful, particularly for surfaces of high roughness.
\end{abstract}

Keywords: ball; disc; surface topography; wear

\section{INTRODUCTION}

Characterization of surface evolution during operating is a substantial topic. Machined surface texture changed during a low wear process, when wear is within a limit of an original surface topography. Change of a surface during wear could be characterized by surface topography measurement. Even if a surface after machining exhibits symmetric roughness, the running-in process often resulted in asymmetric ordinate distribution [1]. The analysis of material ratio curve can give information about wear progress. During a low wear process typically the parameters describing peak surface part, mean amplitude parameters like $S a$ and $S q$ decreased, the skewness $S s k$ decreased and kurtosis $S k u$ increased. In an initial wear stage, only a peak surface part changed, while core and valley parts remained unaltered. Directionality of texture changed, too $[2,3]$.

In order to better understand phenomena occurring during friction, tribological testers were developed. They provide the comprehensive and versatile means of investigating tribology - friction, wear and lubrication. Measuring friction, wear is a way for engineers to understand how materials, coatings and lubricants will stand up to the rigors of various applications. Test procedures must include the ability to reproduce real operating. Manufacturers are searching for improvements and validation of their processes and products.

Surface topography measurement can be used for characterization of types of wear and for microscopic wear measurement. There are various possibilities of wear measurement. In the first case only details of worn surface can be measured without fragments not subjected to wear. One can obtain information about wear amount only after measurement of a worn surface. Wear measurement of balls and discs can be typical examples [4]. In the second case surface topography should be measured in the same place before and after friction test. Initially a mechanical relocation is typically used, utilizing for instance Vicker's indenters $[2,3,5,6]$. After a mechanical treatment software relocation should be used. Many papers reported methods for relocating three-dimensional surface texture accurately, allowing compensation of lateral and rotational dislocations [7-12]. Surface topography measurements performed before and after wear process are typically compared using a cross-correlation method [13] to compensate rotational and translational misalignment.
For linear wear larger than an initial height of surface topography it is necessary to measure simultaneously two parts of surface: subjected and not-subjected to wear [14, $15]$.

\section{MATERIALS AND METHODS}

Surface topographies of discs and balls after wear process were analysed. Discs were made from $42 \mathrm{CrMo} 4$ steel with hardness of $42 \mathrm{HRC}$, but balls from 100Cr6 steel with hardness of $64 \mathrm{HRC}$. Two types of tribological tests were conducted. In the first of them, wear tests were carried out using a ball-on-disc tester, in which a stationary ball was put in contact with a rotated disc under various operating conditions (normal load, speed, sliding distance). The second tests were conducted using a SRV ball-on-disc tester under gross fretting conditions. Comparatively flat spheres ( $R a$ about $0.1 \mu \mathrm{m})$ were used as counter specimens. However disc specimens had different surface topographies obtained after various types of machining (lapping, polishing, grinding, milling and vapour blasting). Surface textures after tribological tests were measured using a white light interferometer Talysurf CCI Lite. Procedures for minimizing errors of wear loss determination will be discussed. They can be applied thanks to the TalyMap software.

\section{WEAR OF DISCS STUDY}

During analysis of surface topography containing a wear scar the first action is to remove form. This is important, because an improper selection of a reference plane can cause a substantial measurement error. For a flat surface only levelling (a polynomial of the first degree) can be used. Application of a polynomial of the second degree can cause a serious distortion of a cross-section of a wear track (see Fig. 1) which can lead to false estimation of disc volumetric wear. It was found that the average errors could be up to $30 \%$. Digital filtering, even by robust Gaussian filter should not be used for the large width of the valley created during wear. When necessary (i.e. substantial waviness occurs) modified filter should be applied [16]. The profile shown in . 1d was determined properly, because areas not subjected to wear from both sides of the wear track were similar (see Fig. 1c). However, in the adverse case, surface levelling can sometimes cause 
distortion of a profile having a worn track. In this case, the best solution is to exclude area of the hole (track) in levelling procedure - see Fig. 2. Generally, errors caused by lack of levelling are higher than those connected with lack of excluding area of scar. The latter errors are larger for larger depths and widths of the scars. When a volume of wear is calculated directly, the errors connected with not proper levelling are very important. However when rotated discs after wear process are analysed, profile levelling can be also used (see Figs. 1 and 2). By proper profile, levelling some errors caused by improper surface levelling can be reduced. For example, application of a polynomial of the second degree can improve substantially profile shown in Fig. 1f. The present authors recommend the use of profile levelling instead of surface levelling in worn profile analysis. a)

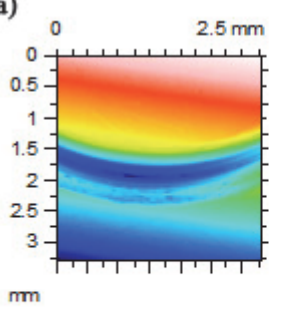

c)

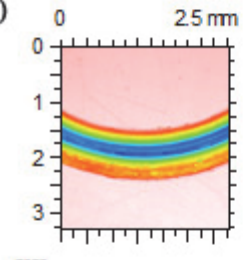

$\mathrm{mm}$

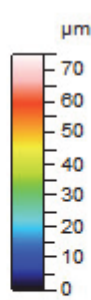

$\mu \mathrm{m}$

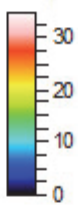

e) 0

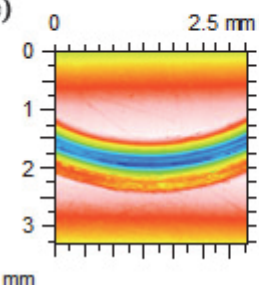

b)

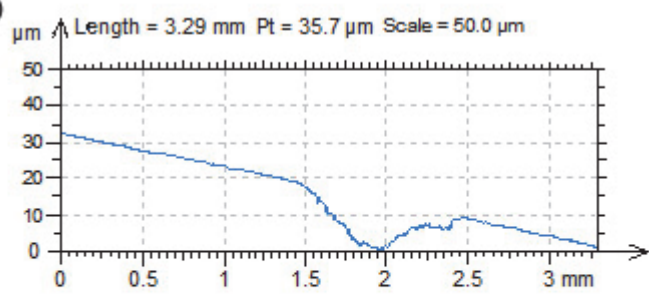

d)

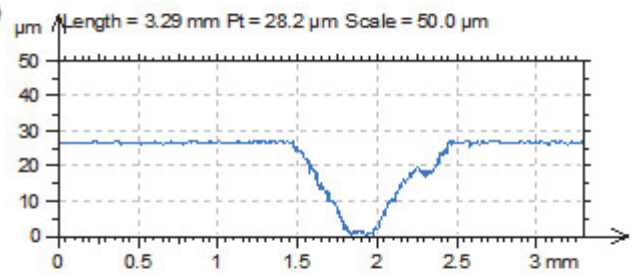

f)

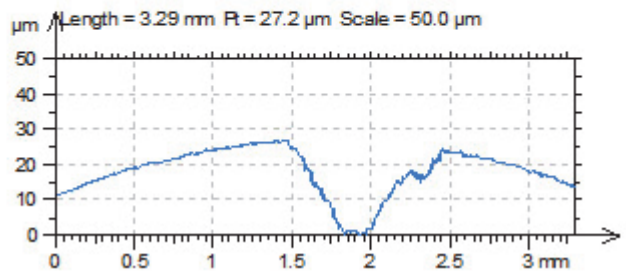

Figure 1 Contour plots of the surface with the wear scar $(a, c, e)$ and cross-sections of the wear scar $(b, d, f)$ of the measured surface $(a, b)$, after form elimination by polynomials of the first $(c, d)$ and the second degree $(e, f)$

a)

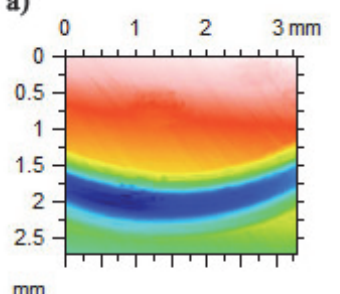

c)

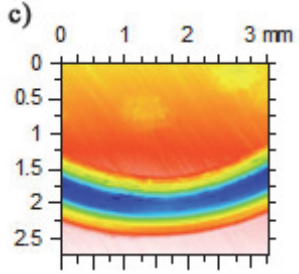

$\mathrm{mm}$

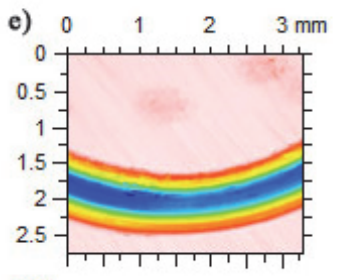

$\mathrm{mm}$
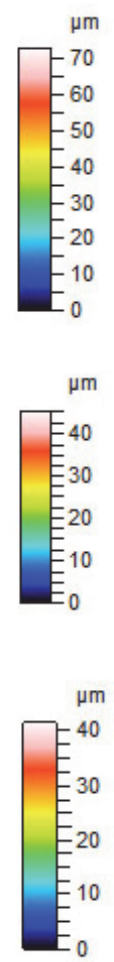

b)

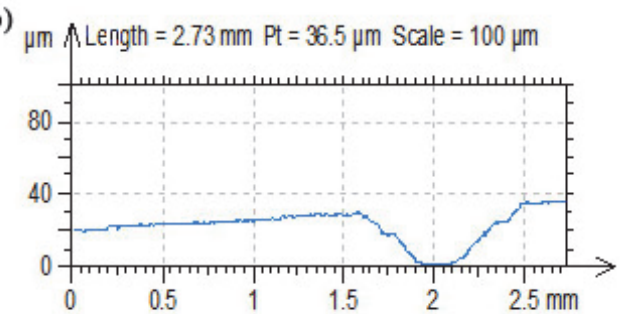

d)
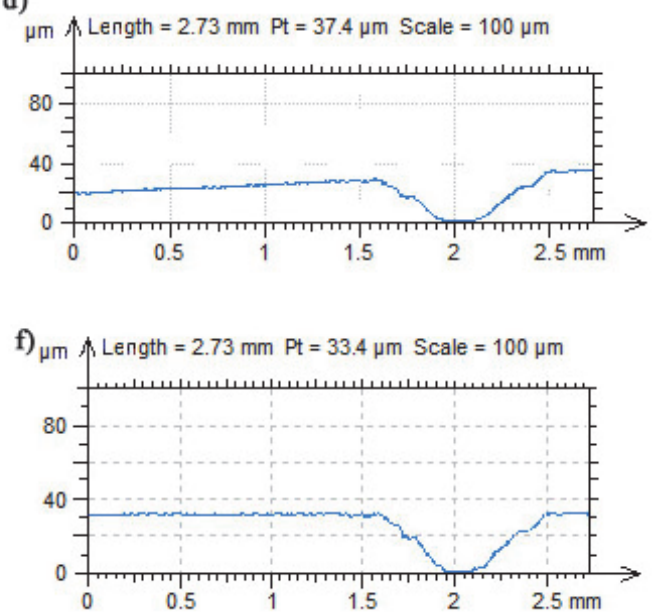

Figure 2 Contour plots of texture with the wear scar $(a, c, e)$ and cross-sections of the wear scar $(b, d, f)$ of the measured surface $(a, b)$, after levelling $(c, d)$ and after levelling excluding the scar area $(e, f)$ 
Similar to areal surface topography analysis when the lengths of profile details not subjected to wear from two sides of groove formed by wear are different, levelling may cause a profile distortion. A profile is typically well levelled, when lengths of its unworn parts from two sides of a groove are similar, even without excluding a wear scar. Truncation of the worn track cross-section (profile zooming) can improve the results (see Fig. 3c). The other and probably the best solution is to exclude the wear scar from levelling (see Fig. 3d). Levelling was done using the least square method.

The distortions caused by improper surface levelling were studied for several worn profiles. It was found that errors caused by lack of levelling led to errors higher than $20 \%$. However lack of scar excluding caused smaller errors, usually up to $7 \%$ in estimation of the hole area. The problem exists when there are several scars on measured profile.

The application of a robust filter is preferred, but when valleys widths are too large, filter should be modified [16]

a)

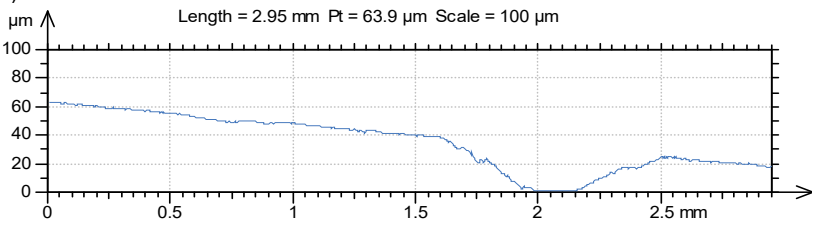

b)

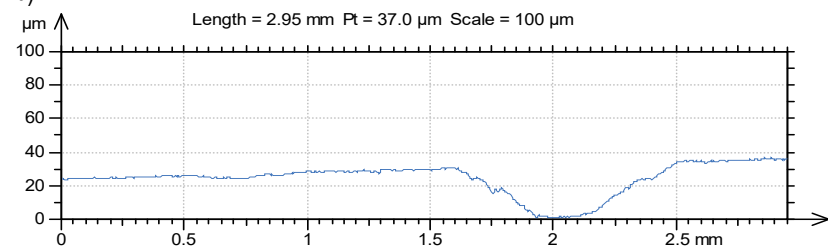

c)

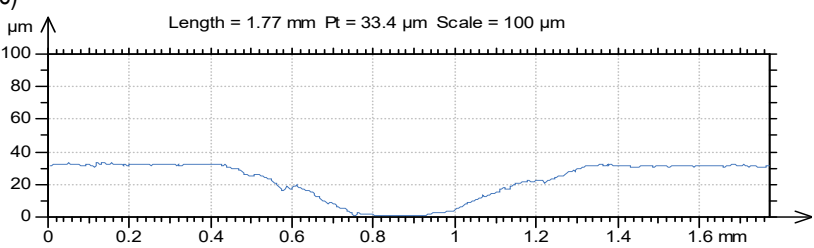

d)

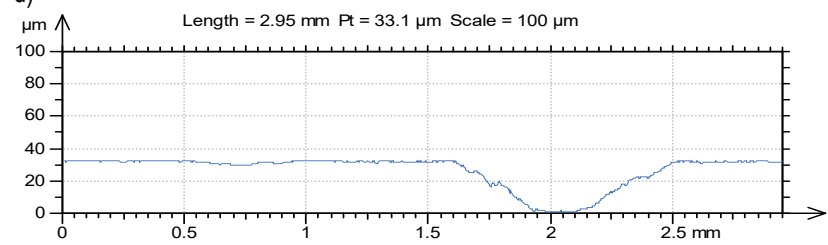

Figure 3 The measured profile (a), the levelled profile (b), the levelled zoomed profile (c), the profile levelled excluding the groove (d)

Typically an area of the hole is computed for wear volume estimation of the rotated disc. The profiles, taken in four positions, $90^{\circ}$ apart, orthogonal to wear track are studied to obtain the wear areas. These areas are eventually averaged and multiplied by the track length. In the case of wear scar examination after fretting and reciprocating motion, volumetric wear can be measured directly. However for quantitative analysis cross sections perpendicular to wear scar can be also studied (see Fig. 4), because the holes sizes changed in the various places. The hole created during wear is assessed by its area, depth and width.

The TalyMap software offers four possibilities of wear sizes computation. In the first (the hole under the mean line) the section under mean line is considered to be the hole, however the section above mean line is considered to be the material that was pushed outside (plastically deformed). The second method (the hole under the water line) looks for the lowest point between the two bars and the highest point. This hole is filled up to the smaller of the two peaks, simulating the filling of the hole using water. The third method (the hole under the peaks line) traces a line between the two peaks from one side to another of the lowest point. The fourth method (the hole between two bars) uses a line between the two bars.

The first method (the hole under the mean line) was found to be the most stable. It is not sensitive to the distance between two bars, unlike the other methods. This distance was changed. It was found that divergences between the results obtained by the first method were negligible (smaller than 1\%). However application of other methods caused deviations up to $20 \%$.

a)

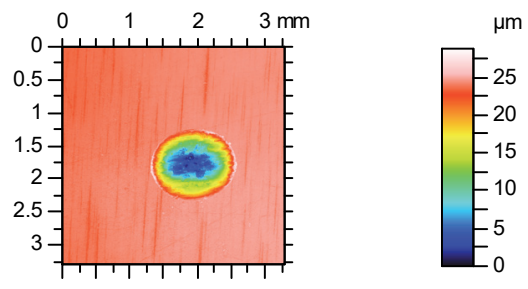

b)

$\mathrm{mm}$

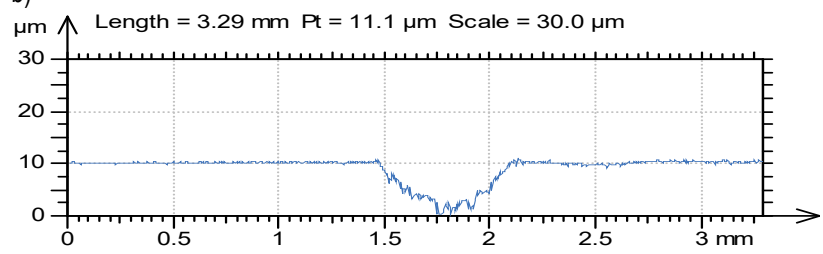

c)

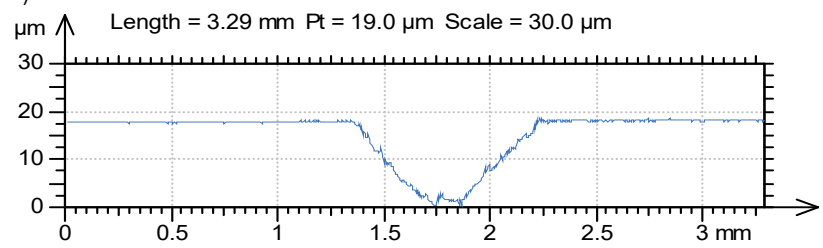

d)

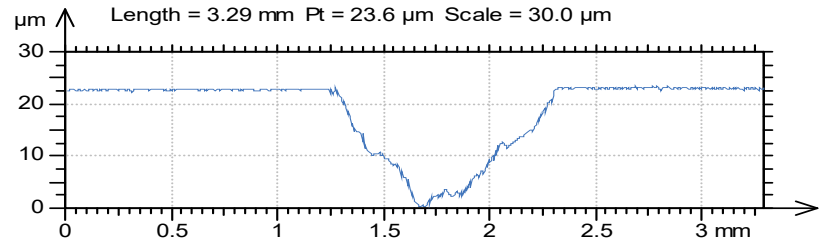

Figure 4 Contour plot of the disc after fretting (a) and cross sections from the left side to the middle of the wear scar $(b, c, d)$

Fig. 5 presents the areas of the hole calculated by the first method (the hole under the mean line) and the second (the hole under the water line). It was found that a small increase in the horizontal distance between bars led to very small deviations when the hole was located under the mean line. However the use of the second method caused relative 
difference of about $3 \%$. These deviations can be larger for rougher disc surfaces.

Generally differences between the first and the other methods were up to $20 \%$. When levelling was done correctly, smooth surfaces were studied and sizes of scars were comparatively large, the relative deviations were small. There is a problem in measuring areas of holes for rough discs and small wear.

It is important to define the reference line for rough surface to calculate the volume below this line [17].

a)

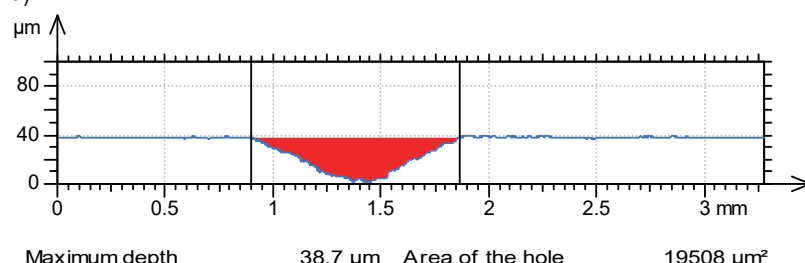

Maximum depth

$38.7 \mu \mathrm{m} \quad$ Area of the hole

$19508 \mu \mathrm{m}^{2}$

b)

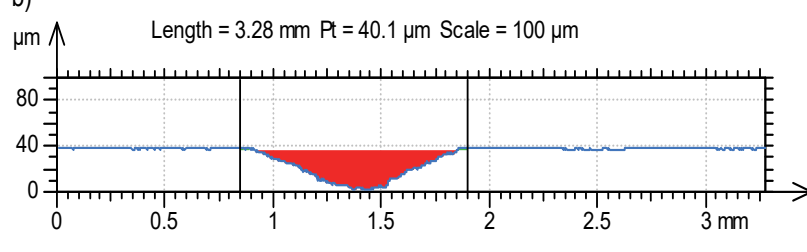

Maximum depth $\quad 38.7 \mu \mathrm{m}$ Area of the hole $\quad 19512 \mu \mathrm{m}^{2}$

c)

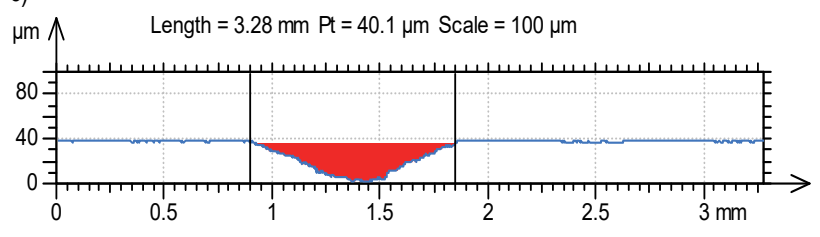

Maximum depth $\quad 38.2 \mu \mathrm{m}$ Area of the hole $\quad 19048 \mu \mathrm{m}^{2}$

d)

$\mu \mathrm{m} \uparrow \quad$ Length $=3.28 \mathrm{~mm} \mathrm{Pt}=40.1 \mu \mathrm{m} \mathrm{Scale}=100 \mu \mathrm{m}$

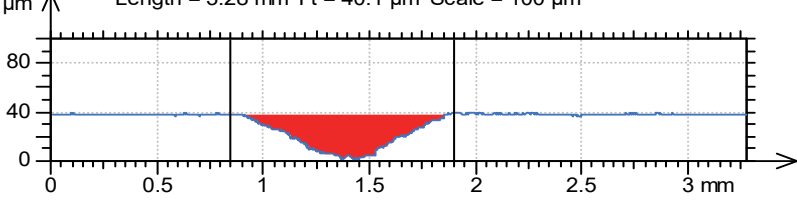

Maximum depth $\quad 38.8 \mu \mathrm{m}$ Area of the hole $\quad 19616 \mu \mathrm{m}^{2}$

Figure 5 The effect of distance between two bars on the area of the hole: $a, c-$ smaller distance, $b, d$ - larger distance, $a, b$ - the hole was located under the mean line, $c, d-$ the hole was located under the water line

In this aspect, a location of the hole under mean line is superior to the other methods. The mean line is calculated for the profile points outside the bars. There can be additional source of deviations of the hole area estimation. Rough surface is typically non stable (different in various places). The mean line is positioned correctly if the profile length outside the bars is comparatively long. When this length is short, only points near the scar affect the hole area estimation. One can see that profile zooming caused an increase in the hole area by more than $10 \%$ (see Fig. 6). This was because in the zoomed detail (Fig. 6c) the left part of the profile did not contain valleys and owing to it the mean line raised.

It is possible to obtain information about types of wear on the basis of worn profile analysis. However, the method based on the mean line can give us also quantitative information about an area of material pushed outside in the case of a material transfer (Fig. $7 \mathrm{a}$ and $7 \mathrm{~b}$ ) and plastic deformation (Fig. 7c). This method also behaves well for analyses of surfaces after scratch tests.

a)

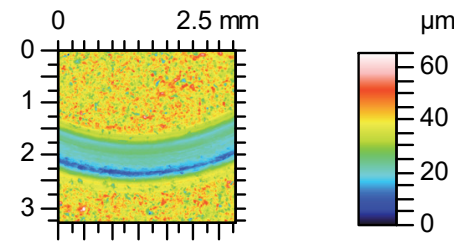

$\mathrm{mm}$

b)

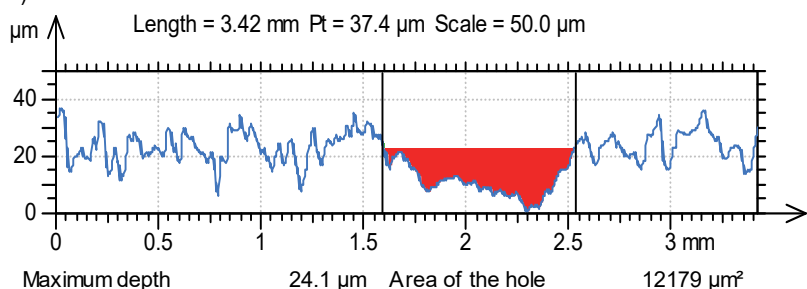

Maximum depth $\quad 24.1 \mu \mathrm{m}$ Area of the hole $\quad 12179 \mu^{2}$

c)

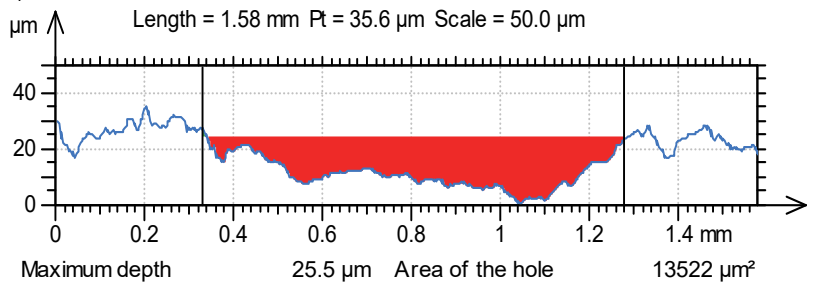

Figure 6 Contour plot of the worn surface (a) and the area of the hole for the measured (b) and the zoomed profile (c)

a)

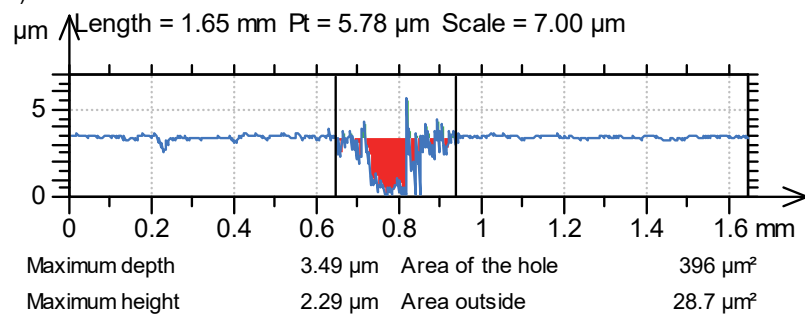

b)

$\mu \mathrm{m} \bigwedge$ Length $=3.07 \mathrm{~mm} \mathrm{Pt}=11.0 \mu \mathrm{m}$ Scale $=12.0 \mu \mathrm{m}$

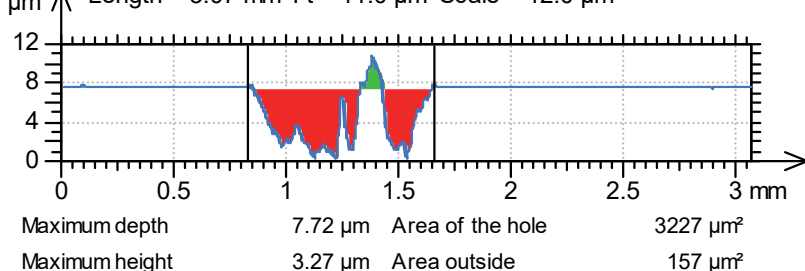

c)

$\mu \mathrm{m} \bigwedge$ Length $=0.769 \mathrm{~mm} \mathrm{Pt}=2.53 \mu \mathrm{m}$ Scale $=3.00 \mu \mathrm{m}$

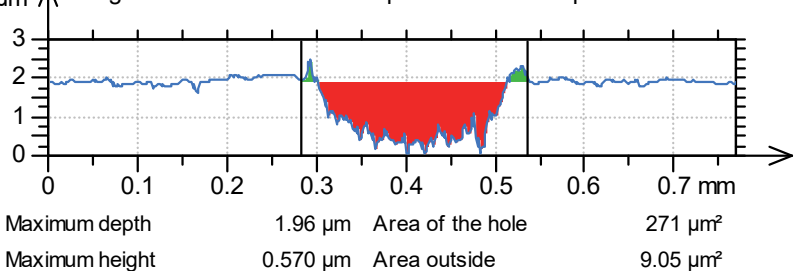

Figure 7 The hole area and the area outside for the material transfer $(a, b)$ or the plastic deformation (c)

Generally, calculation of the hole area under the mean line is recommended by the present authors. However, in special cases (for example in computation of a dimple area) the hole can be located under the water line. 
The analysis of the worn disc can give us additional information about the kind of contact. When a ball co-acted a disc typically during wear a non-conformal (point) contact changes into conformal, due to wear of a ball. When there are difficulties in ball measuring worn disc can be source of information about type of contact.

The radius of the ball in experiment was $3.175 \mathrm{~mm}$. Because it was found that the curvature of the hole was removed using the circle of similar diameter $(3.23 \mathrm{~mm})$ one can conclude that the contact was non-conformal during the test (see Fig. 8).

When the volumetric wear is calculated directly, four methods of its computation are available. In the method called the least square plane, the volume is calculated between the bottom of the hole and the least square plane. This method, similar to the mean line method in profile analysis can be used for surfaces after form removal. Method called is similar but the polynomial surface is used instead of the least square plane. It was found that application of a polynomial of the second degree gave similar results to application of the least square plane. Differences were smaller than 5\%. In vertical lines method the volume is calculated column by column, considering 2 points situated under the edge of the hole. The horizontal lines method is similar but calculated per lines. Two last mentioned methods should be used for not flat (mainly cylindrical) surface.

a)

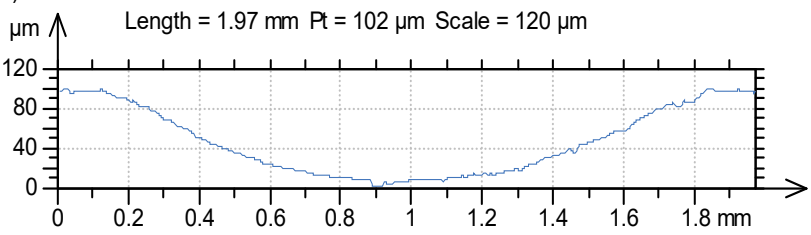

b)

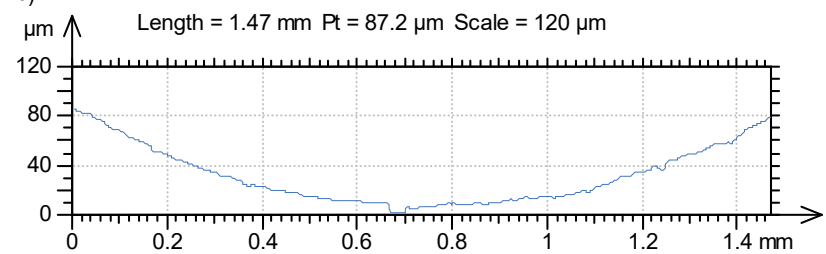

c)

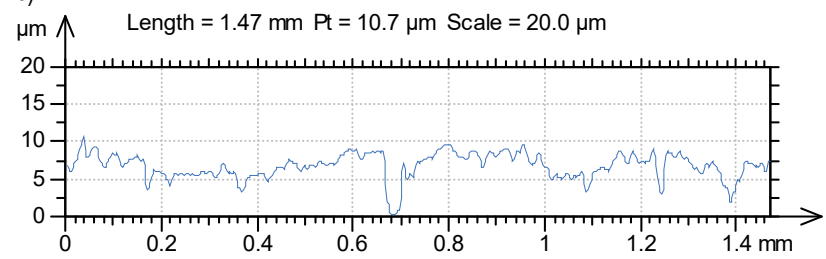

Figure 8 Profile with the wear scar (a), the zoomed profile (b) and the zoomed profile after form removal using a circle (c)

These methods were tested for the disc surfaces after wear and the least square method was recommended. It can be used also for a curved surface after proper form removal. After application of this method not only the volume of the hole but also the volume of the peak can be calculated, which is important in the cases of the material transfer or the plastic deformation. When the hole was created as the results of fretting, various wear measures exist, not only areas of holes, their depths and widths on various cross-sections (Figure 4) but also the horizontal area, the volume of the surface and the maximum depth of the hole, therefore proper calculations of these parameters are very important. It was found that application of methods based on horizontal and vertical lines led typically to overestimation of the scar volume compared to the least square plane method up to $20 \%$ when the contour was correctly determined. The least square plane method is also more stable than methods based on lines - see Fig. 9. For various contours, application of horizontal lines method caused large changes of the hole volume, however these changes were negligible after application of the least square method: the following volumes were obtained: 10639689 and $10651662 \mu^{2}$. Reasonable change of contour can lead to change of the scar volume to $20 \%$ when horizontal or vertical lines methods were used.

However, similar to profiles, estimation of wear volume is not stable for a comparatively rough surface even if the least square plane was used. In order to proper compute this volume the measurement area outside the hole ought to be comparatively large. A decrease in this area caused changes of wear volume about $6 \%$ (see Fig. $10)$.

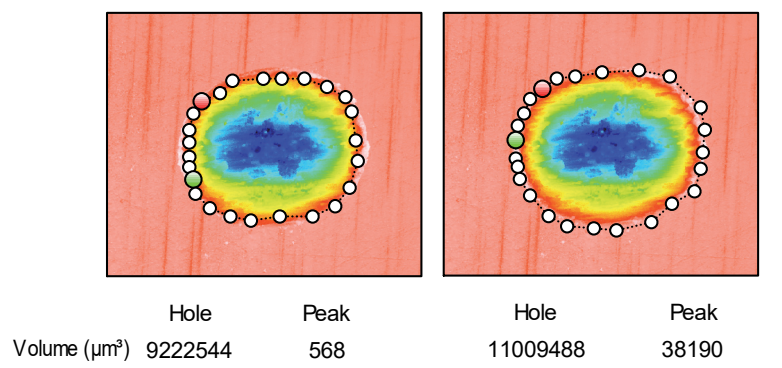

Figure 9 Hole volume calculated by the horizontal line method for various contours of the wear scar

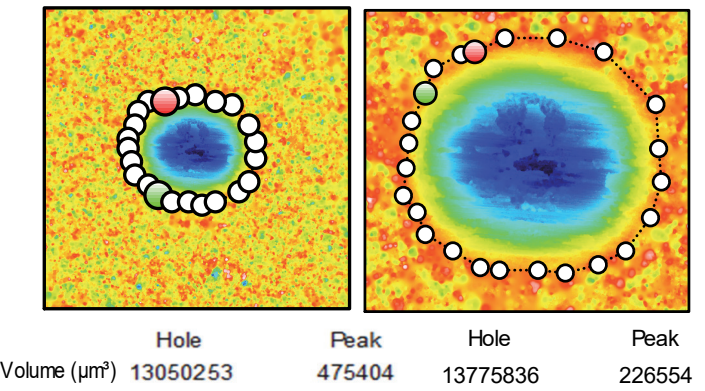

Figure 10 Change of the hole volume for various measurement areas

\section{ANALYSIS OF WEAR OF BALLS}

In the wear of balls estimation a sphere must be used for form removal. The TalyMap software offers this possibility. It is important to exclude the wear scar. After form removal one should check the radius of the sphere. It should be close to the nominal radius. Similarly to worn discs, the least square plane method is recommended for worn balls. Fig. 11 shows the contour plot and the isometric view of the worn ball before form removal as well as the results of wear measurement.

To analyse wear of the cross-section of the ball, two methods may be used. In the first of them, form should be removed using the sphere and then profile can be extracted (see Fig. 12a, 12b and 12c). 
a)

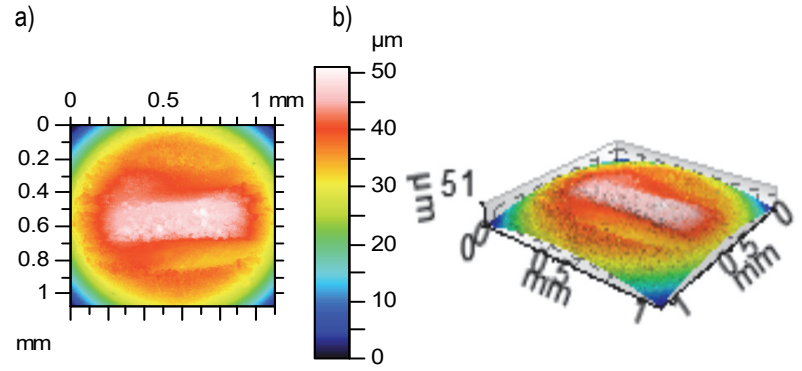

c)

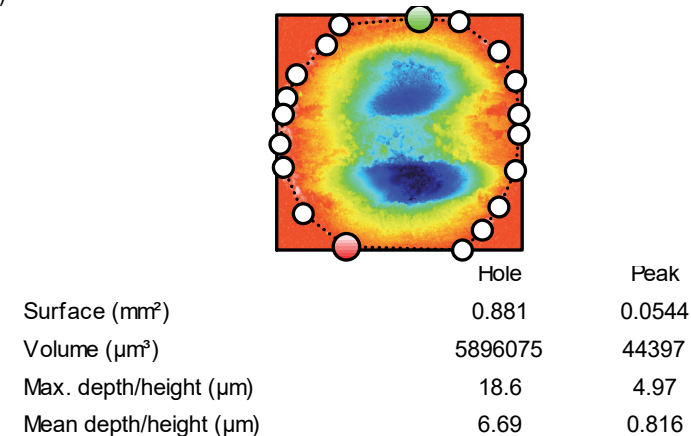

Figure 11 The contour plot (a) and the isometric view (b) of the wear scar of the ball before form removal and the results of wear measurement after form removal by a sphere

a)

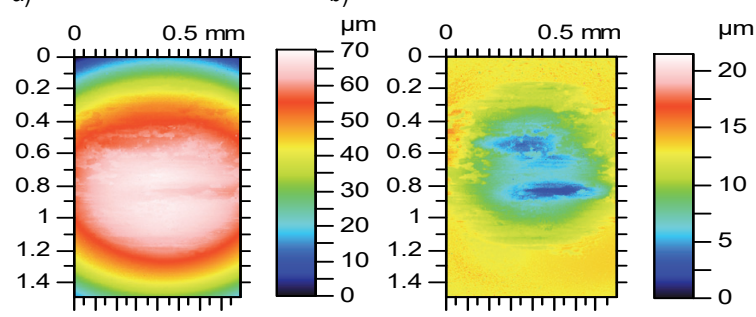

$\mathrm{mm}$

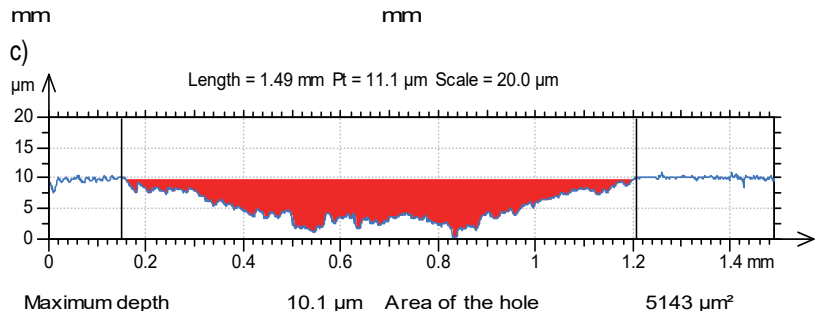

d)

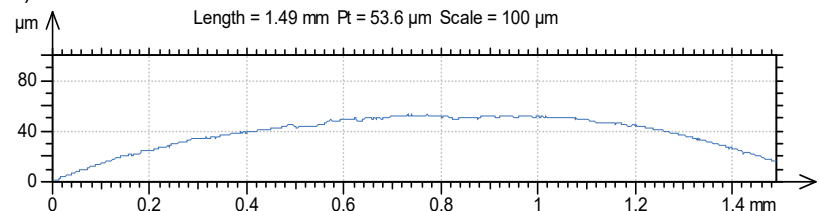

e)

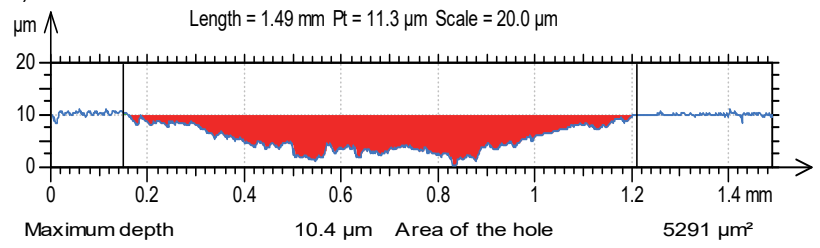

Figure 12 Contour plot of the worn ball before (a) and after form removal using a sphere (b), the extracted profile of the worn ball after form removal (c), the profile before (d) and after form removal using a circle (e)

In the second method profile can be extracted from initial surface and then form should be removed using circle (see Fig. $12 \mathrm{~d}$ and 12e). The first method is recommended because the correctness of form removal can be checked by comparison of nominal ball radius with radius of sphere used in the form removal. Furthermore it is easier to select a profile after the form removal. However the second method should be used when it is difficult to apply the first method. These two methods can give different results even when the same profile is analysed see Fig. 12. The hole under the mean line method should be used in the area of the wear scar calculation.

Similar to disc analysis it is possible to assess the kind of wear or to obtain quantitative information about the material transfer.

The analysis of worn ball is also helpful in the assessment of a contact type. When wear of the ball is larger in its middle part, the non-conformal contact changed into conformal during wear - see Fig. 12.

However when the wear amount is similar across the wear track of the ball the contact is conformal during the test, see Fig. 13, which presents the contour plot and the profile of the worn ball co-acted with the disc, which profile is shown in Fig. 8.

a)

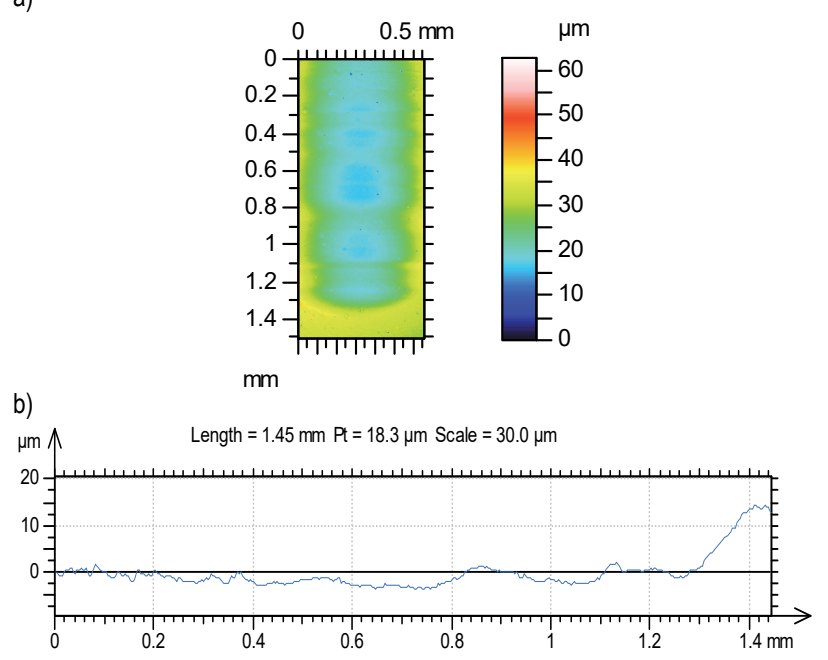

Figure 13 The contour plot (a) and the profile (b) of the worn ball detail after form removal

\section{CONCLUSIONS}

Application of wear analysis of discs and balls on a micro scale allows us for precise determination of their wear levels only after tribological test, however this analysis should be careful. Profile levelling of disc is necessary before the wear measurement. The lack of levelling can lead to errors larger than $20 \%$. The hole should be excluded from the levelling. The lack of wear scar excluding can cause errors up to $7 \%$. The effect of wear scar excluding is smaller when the hole is located in the middle part of the profile. In areal (3D) surface levelling the hole should be also excluded.

The area of the hole should be calculated under the mean line but the volume under the mean plane. Application of these methods can lead to estimation of not only wear of holes but also material transfers or plastic deformations. Calculated scar sizes using these techniques are stable. Application of the other methods can lead to errors in quantitative wear estimation typically up to $20 \%$. These techniques, contrary to the least square methods are sensitive to the width or the contour of the wear scar. The 
use of a polynomial of the second degree gave similar results to application of the least square plane (relative errors are smaller than $5 \%$ ).

The small errors of wear volume calculation are possible for the large measuring length (area), the measurement of smooth surfaces and large sizes of scars.

The measurement of rough textures is very sensitive to errors. In this case the use of the least square methods is strongly recommended. However after its application the relative deviations can be up to $7 \%$ for different measuring length/area.

In estimation of the wear of balls, the form should be removed by application of a sphere or a circle. Correctness of form removal should be checked by comparing the applied radius with the nominal radius of ball. In profile analysis, form removal of the areal surface is recommended.

It is possible to check the kind of a contact (conformal or non-conformal) and the type of wear after analyses of worn discs and balls.

\section{REFERENCES}

[1] Leefe, S. E. (1998). "Bi-Gaussian" representation of worn surface topography in elastic contact problems. Tribology for Energy Conservation. Proceedings of the $24^{\text {th }}$ LeedsLyon Symposium on Tribology / London, 281-290. https://doi.org/10.1016/S0167-8922(98)80083-7

[2] Grabon, W., Pawlus, P., \& Sep, J. (2010). Tribological characteristics of one-process and two-process cylinder liner honed surfaces under reciprocating sliding conditions. Tribology International, 43(10), 1882-1892. https://doi.org/10.1016/j.triboint.2010.02.003

[3] Grabon, W., Koszela, W., Pawlus, P., \& Ochwat, S. (2013). Improving tribological performance of piston ring - cylinder liner frictional pair by liner surface texturing. Tribology International, 61, 102-108. https://doi.org/10.1016/j.triboint.2012.11.027

[4] Dzierwa, A., Pawlus, P., Zelasko, W., \& Reizer R. (2013) The study of the tribological properties of one-process and two-process textures after vapour blasting and lapping using pin-on disc tests. Key Engineering Materials, 527, 217-222. https://doi.org/10.4028/www.scientific.net/KEM.527.217

[5] Pawlus, P., Galda, L., Dzierwa, A., \& Koszela, W. (2009). Abrasive wear resistance of tectured steel rings. Wear 267(11), 1873-1882. https://doi.org/10.1016/j.wear.2009.03.003

[6] Rosen, B.-G. (1994). Towards surface engineering. PhD Dissertation. Chalmers University of Technology, Gothenburg.

[7] Cabanettes, F., Claret-Tournier, J., Mohlin, J., Nilsson, P. H., Rosen, B. G., \& Xiao, L. (2009). The evolution of surface topography of injection cams. Wear, 266(5-6), 570-573. https://doi.org/10.1016/j.wear.2008.04.057

[8] Gahlin, R. \& Jacobson, S. (1998). A novel method to map and quantify wear on a micro-scale. Wear, 222(2), 93-102. https://doi.org/10.1016/S0043-1648(98)00287-7

[9] Sasajima, K., Naoi, K., \& Tsukada, T. (2000). A softwarebased relocation technique for surface asperity profiles and its application to calculate volume changes in running-in wear. Wear, 240(1-2), 152-163. https://doi.org/10.1016/S0043-1648(00)00347-1

[10] Balcon, M., Marinello, F., Carmignato, S., \& Savio, E. (2011). Wear analysis through surface relocation. Proceedings of $13^{\text {th }}$ International Met $\&$ Props Conference, Twickenham Stadium UK, 316-319. https://doi.org/10.1088/1742-6596/311/1/012020
[11] Condeco, J., Christensen, L. H., \& Rosen, B.-G. (2001). Software relocation of $3 \mathrm{D}$ surface topography measurements. International Journal of Machine Tools and Manufacture, 41(13-14), 2095-2101. https://doi.org/10.1016/S0890-6955(01)00076-1

[12] Zelasko, W., Pawlus, P., Dzierwa, A., \& Prucnal, S. (2016). Experimental investigation of plastic contact between a rough steel surface and a flat sintered carbide surface. Tribology International, 100, 141-151. https://doi.org/10.1016/j.triboint.2015.12.015

[13] Vorburger, T. V., Song, J.-F., Chu, W., Ma, L., Buu, S. H., Zheng, Z., \& Renegar, T. B. (2011). Application of crosscorrelation function. Wear, 271(3-4), 529-533. https://doi.org/10.1016/j.wear.2010.03.030

[14] Cho, Y.-J., Koo, Y.-P., \& Leon, J.-H. (2002). Surface profile estimation by digital filtering for wear volume calculation. Wear, 252(3-4), 173-178. https://doi.org/10.1016/S0043-1648(01)00836-5

[15] Sep, J., Pawlus, P., \& Dzierwa, A. (2013). The analysis of abrasive wear of the helical grooved journal bearings. Key Engineering Materials, 527, 173-178. https://doi.org/10.4028/www.scientific.net/KEM.527.173

[16] Dobrzański, P. \& Pawlus, P. (2013). Modification of robust filtering of stratified surface topography. Metrology and Measurement Systems, 20(1), 107-118. https://doi.org/10.2478/mms-2013-0010

[17] Kubiak, K. J., Liskiewicz, T. W., \& Mathia, T. G. (2011). Surface morphology in engineering applications: Influence of roughness on sliding and wear in fretting. Tribology International, 44(11), 1427-1432. https://doi.org/10.1016/j.triboint.2011.04.020

\section{Contact information:}

\section{Pawel PAWLUS, Professor}

Rzeszow University of Technology

Faculty of Mechanical Engineering and Astronautics

Powstancow Warszawy 8 Av., 35-959 Rzeszow, Poland

E-mail: ppawlus@prz.edu.pl

Andrzej DZIERWA, Mech. Eng. PhD

Rzeszow University of Technology

Faculty of Mechanical Engineering and Astronautics

Powstancow Warszawy 8 Av., 35-959 Rzeszow, Poland

E-mail: adktmiop@prz.edu.pl 